(Review Article)

\title{
APPLICATIONS OF HAHN-BANACH PRINCIPLE TO THE MOMENT PROBLEM
}

\author{
Octav Olteanu
}

\author{
Date of Receiving : $\quad$ : 14.11.2014 \\ Date of Acceptance : 14.02 .2015
}

\begin{abstract}
The first aim of this review paper is to show how Hahn-Banach type results can be applied in several aspects related to the multidimensional real moment problem. Here the main tools are constrained extension theorems for linear functionals or operators. An application using suitable $L^{1}$-polynomial approximation on unbounded subsets is also discussed. One uses approximation by sums of tensor products of positive polynomials in each separate variable. This way, one solves the difficulty created by the fact that there are positive polynomials, which are not writable as sums of squares in several dimensions. One characterizes the existence of a solution in terms of computable quadratic forms, similarly to the one-dimensional case. This is the second aim of the paper. The third aim consists in proving applications related to the connection of the moment problem with the extreme "points" and some inverse problems, similar to those solved in the literature by some other methods. Remarks on the multiplicative solutions of some Markov moment problems are recalled in the end of this work.
\end{abstract}

\section{Introduction}

The first part of this paper recalls some results on the extension of linear operators, usually applied in solving moment problems. The main tools are constrained extension theorems for linear functionals or operators. The upper constraint measures the continuity and the norm of the solution. The lower constraint is usually the positivity of the solution on the positive cone of the domain - space. Possible representation by means of a positive scalar or vector measure works for such a solution. Applications to the moment problem are deduced in Section 4. This is the first aim of the paper. On the other hand, we point out the importance of approximation methods in several dimensions, due to the fact that the "form" of positive polynomials on simple unbounded subsets is unknown. Polynomial decomposition and approximation in studying the existence and uniqueness of the solutions of some moment problems have been studied in [1] - [5], [7], [8], [11]-[15], [18]-[24], [26]. Finding explicit

2010 Mathematics Subject Classification. 47A57, 47A50, 41A10.

Key words and phrases. Hahn-Banach principle, moment problem, approximation, concrete spaces.

Communicated by. Shiv Kumar Kaushik 